\title{
AJUSTE DE MODElos de PLATÔ DE RESPOSTA PARA A EXIGÊNCIA DE ZINCO EM FRANGOS DE CORTE ${ }^{1}$
}

\author{
Fitting response plateau models to $\mathrm{Zn}$ requirements in broilers
}

\author{
Douglas Messias Lamounier Camargos Rezende ${ }^{2}$, Joel Augusto Muniz ${ }^{3}$, Daniel Furtado Ferreira ${ }^{4}$, \\ Fabyano Fonseca e Silva ${ }^{5}$, Luiz Henrique de Aquino ${ }^{6}$
}

\begin{abstract}
RESUMO
Com este trabalho, objetivou-se estudar a técnica platô de resposta utilizando modelos de regressão segmentada para avaliar sua aplicação no estudo da exigência de $\mathrm{Zn}$ em frango de corte. Foram utilizados os dados de uma pesquisa com frangos de corte envolvendo um experimento fatorial 2 × 2 x 9, dois experimentos, dois sexos e nove doses de zinco na ração, considerando 8 repetições. As doses de $\mathrm{Zn}$ estudados foram: 0, 15, 30, 45, 60, 75, 90, 105, $120 \mathrm{ppm}$. A variável avaliada foi o teor de Zn na tíbia em ppm. Foram utilizadas rotinas no procedimento não-linear (PROC NLIN do SAS ${ }^{\circledR}$ ) para estimação dos parâmetros, utilizando-se as médias da variável resposta nas diversas doses. Dois modelos não-lineares (MNLE1 e MNLE2) e o Modelo Polinomial Quadrático (MPQ), ambos com platô, foram comparados. Os três modelos foram estatisticamente equivalentes, contudo o modelo MNLE1 mostrou-se mais adequado devido a sua maior facilidade de interpretação dos parâmetros.
\end{abstract}

Termos para indexação: Aves de corte, modelos não-lineares, platô de resposta.

\section{ABSTRACT}

This work had for objective to apply the response plateau technique to evaluate the methodology and the models in the requirements of $\mathrm{Zn}$ in broiler. The experiment was a completely randomized design with $(2 \times 2 \times 9)$ factorial structure, being 2 experiments, 2 Sex (sexes) and 9 levels of $\mathrm{Zn}$, with 8 replications. The levels of $\mathrm{Zn}$ were: 0, 15, 30, 45, 60, 75, 90, 105, 120 ppm. The studied variable was $\mathrm{Zn}$ level in the tibia in ppm. The models were fitted using the averages of the doses from the initial analysis of variance in SAS PROC NLIN (SAS ${ }^{\circledR}$ ). Two nonlinear models (MNLE1 and MLNE2) and the quadratic polinomial model (MPQ), both with plateau, were compared. The three models results equivalent fitting to data. As MNLE1 has the easiest interpretation it was sugested as the best model.

Index terms: Broilers, nonlinear models, response plateau.

\section{(Recebido em 13 de janeiro de 2004 e aprovado em 26 de setembro de 2005)}

\section{INTRODUÇÃO}

Com o constante melhoramento genético das aves, as linhagens de frangos de corte têm adquirido capacidade de expressar cada vez mais rápido seu alto potencial produtivo. Porém, esse potencial só é eficientemente aproveitado, quando se tem, além de outros fatores, fornecimento adequado de todos os nutrientes exigidos. Por isso, várias pesquisas têm sido desenvolvidas para determinar suas exigências nutricionais em diferentes idades, sexo e linhagens, com o objetivo de obter alimentação de menor custo, que permita o máximo aproveitamento do potencial genético da ave (RUNHO et al., 2001).

De acordo com Euclydes \& Rostagno (2001), a adição de um nutriente limitante na ração, mantendo níveis adequados dos demais nutrientes, resultará, em crescimento do animal até que sua exigência seja atendida. A partir daí, existirá uma faixa de estabilização no crescimento e, em seguida, dependendo do nutriente que está sendo adicionado, poderá ocorrer uma perda de peso do animal. Os autores apresentam o esquema ilustrativo do efeito de um nutriente limitante (Figura 1), em que se observa que o fenômeno resultante do acréscimo de um nutriente na ração, partindo de níveis baixos até níveis elevados, pode ser descrito em quatro fases:

i. Inicial - Fase em que o acréscimo do nutriente garante apenas a sobrevivência do animal, pois os níveis são insuficientes para permitir o crescimento;

ii. Resposta - Fase na qual os animais começam a apresentar crescimento, melhor eficiência alimentar, entre

Parte da dissertação de apresentada à UFLA pelo primeiro autor para obtenção do título de Mestre em Agronomia/Estatística e Experimentação Agropecuária. Apoio financeiro do CNPq.

${ }^{2}$ Professor Adjunto I de Estatística da UNIPAC/Bom Despacho - Bom Despacho, MG - dougmes@yahoo.com.br

${ }^{3}$ Professor Titular do Departamento de Ciências Exatas da Universidade Federal de Lavras/UFLA - Cx. P. 3037 - $37200-000$ - Lavras, MG Bolsista CNPq - joamuniz@ufla.br

${ }^{4}$ Professor Adjunto III do Departamento de Ciências Exatas da Universidade Federal de Lavras/UFLA - Cx. P. 3037 - $37200-000$ - Lavras, MG danielff@ufla.br

${ }^{5}$ Professor de Estatística do Departamento de Informática da Universidade Federal de Viçosa/UFV - fabyano@dpi.ufv.br

${ }^{6}$ Professor do Departamento de Ciências Exatas Universidade Federal de Lavras/UFLA - Cx. P. 3037 - 37200-000 - Lavras, MG. 
outras vantagens, até um nível em que se estabiliza a desempenho;

iii. Estável - Fase em que os níveis do nutriente não promovem resposta ao desempenho;

iv. Tóxica - Fase em que o nível elevado do nutriente pode causar redução no desempenho em conseqüência de efeitos colaterais.

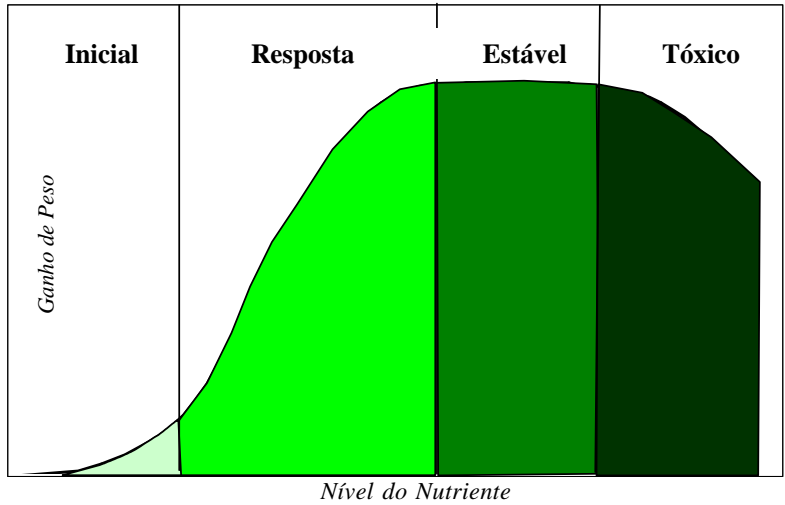

FIGURA 1 - Curva de resposta dos animais à adição de um nutriente limitante à ração.

Os autores consideram, ainda, que os modelos utilizados para determinar os níveis ótimos dos nutrientes na ração são aplicáveis quando os experimentos são conduzidos nas fases "Resposta" e "Estável". Se o experimento for instalado somente no começo da fase "Resposta", o nível ideal do nutriente poderá não ser determinado, porque a resposta máxima não foi atingida. Se o experimento tiver início no decorrer da fase "Estável", concluir-se-á que o nutriente em estudo não é essencial ou está acima da exigência. Caso os níveis estudados se situem na fase "Tóxica", o resultado mostrará efeito nocivo aos animais.

Experimentos do tipo Dose $\mathrm{x}$ Resposta são muito comuns em várias áreas da experimentação. Eles são utilizados para obter os níveis recomendáveis dos minerais nas formulações de rações para animais, para avaliar níveis de proteína, energia e outros ingredientes em rações animais de diferentes espécies e estudar a conversão alimentar, bem como no estudo das formulações de adubos para plantações cultivadas em diferentes solos. Para estes experimentos, entre as técnicas de análises estatísticas que podem ser utilizadas para descrever os níveis ótimos de um nutriente, destacam-se o modelo polinomial quadrático, broken line, Platô linear, Platô quadrático e Platô nãolineares.

\section{i. O Modelo Broken Line}

Segundo Coelho et al. (1987), para a análise de exigências nutricionais dos animais um dos modelos utilizados é o de linha quebrada, ou broken line. Baker (1986) e Robbins et al. (1979) avaliaram que este modelo combina um teste de média para determinação do platô e ajusta uma equação linear aos dados que apresentam respostas a níveis crescentes de nutrientes. A exigência é dada no ponto em que uma perpendicular baixada da intersecção da reta com o platô encontrar o eixo da abscissa. De acordo com Pack (1996), o modelo de regressão linear mais usado em estudos de nutrição animal é o modelo broken line, que apesar de apresentar um ajustamento aceitável, não é fisiologicamente o mais adequado.

\section{ii. O modelo platô de resposta linear (PRL)}

Este modelo platô de resposta linear foi aplicado inicialmente em ensaios sobre fertilidade do solo (BRAGA, 1983). Sua principal vantagem é que na presença de doses muito elevadas de algum elemento torna-se possível estimar a diminuição de desempenho ocasionada pelo excesso. Segundo Coelho et al. (1987), o modelo descontínuo PRL permite a comparação de combinações alternativas de retas e platô permitindo escolher como melhor opção de ajustamento a combinação que tenha apresentado a menor soma de quadrados dos desvios. À semelhança do broken line, a exigência em determinado nutriente é dada no ponto em que a perpendicular baixada da intersecção da reta com o platô encontrar a abscissa. As respostas nem sempre apresentam esta configuração em virtude do complexo sistema fisiológico do animal. Morris (1983) e Runho et al. (2001) afirmam que o ajuste de dados pelo modelo PRL em casos especiais proporciona bom ajuste estatístico, mas freqüentemente subestima à dose ótima. Vários autores utilizaram o modelo PRL em pesquisas com diferentes espécies na área de Zootecnia: em frangos de corte, Cella et al. (2001) e Gomes et al. (1994); em poedeiras, Figueiredo et al. (2001); em suínos, Coelho (1984) e Oliveira et al. (2001), entre outros. A maior parte destes trabalhos foi desenvolvida na UFV, utilizando como suporte estatístico o programa SAEG, cuja grande versatilidade serviu para difundir o uso desse modelo.

\section{iii. $\mathrm{O}$ modelo polinomial quadrático}

O modelo quadrático ( $\left.y=a+b x+c x^{2}\right)$, sobre o ponto de vista "biológico", é mais utilizado que o modelo linear por possibilitar a determinação do ponto de máximo. Todavia, dependendo da natureza dos dados e do 
experimento, esse modelo pode não ser adequado. Morris (1983) e Runho et al. (2001) afirmam que o uso do Modelo Polinomial Quadrático em algumas situações não proporciona bom ajuste dos dados, causando falsa segurança, uma vez que a curvatura é muito sensível a variações nos intervalos dos tratamentos e o modelo pode não ser fisiologicamente correto, pois pressupõe respostas simétricas para a deficiência e para o excesso de nutrientes.

\section{iv. O Modelo Polinomial Quadrático com platô}

O procedimento não-linear do software Statistical Analysis System, (SAS INSTITUTE, 1995), apresenta um exemplo da aplicação da técnica platô de resposta quadrático. Neste exemplo, o ponto de início do platô foi denominado de $x_{0}$, que é localizado por meio da primeira derivada da equação quadrática, ou seja, pelo ponto de máximo. Quando $x$ é maior que $x_{0}$, este ponto se torna uma constante, transformando-se numa reta com inclinação zero, denominada platô, cujo modelo é dado por $y=p$; e quando $x$ é menor que $x_{0}$, este ponto se encontra na parte quadrática do modelo ( $\left.y=a+b x+c x^{2}\right)$ sendo descrito por uma parábola. Para que esta técnica possa ser aplicada, exigese que a curva seja contínua e suave. Bullock $\&$ Bullock (1994), Carrijo \& Hochmuth (2000) e Cerrato \& Blackmer (1990), utilizaram esta técnica, observando um bom comportamento do modelo na descrição das respostas em experimentos na área de Ciências Agrárias.

\section{v. Modelos não-lineares}

Neste caso a técnica platô de resposta é utilizada conjugada com o ajuste de modelos não-lineares, conforme o trabalho de Rezende el al. (2000), que desenvolveram um estudo com dados de nutrição animal. Euclydes \& Rostagno (2001) comentam que parece não existir uma metodologia adequada para análise de dados no caso de ajuste de modelos de resposta para nutrientes. Segundo os autores, os diversos pesquisadores têm simplesmente se limitado a apresentar críticas às metodologias de ajuste dos modelos existentes, sem propor o melhor modelo. Morris (1983) considera que os métodos estatísticos de estimação e ajuste de modelos, apesar de serem bastante lógicos, nem sempre apresentam conclusões apropriadas.

Este trabalho teve por objetivo estudar a técnica platô de resposta utilizando modelos de regressão segmentada para avaliar sua aplicação no estudo da exigência de $\mathrm{Zn}$ em frango de corte.

\section{MATERIAL E MÉTODOS}

O material experimental utilizado corresponde a pintos de um dia da linhagem Hubbard, sexados, vacinados contra as doenças Marek e Bouba Aviária. Foram alojados inicialmente 12 pintos do mesmo sexo por box e no final da primeira semana feita uma refugagem, reduzindo o total para 10 pintos. A partir do primeiro dia, as aves receberam as dietas experimentais e água deionizada à vontade.

No $27^{\circ}$ dia de idade selecionou-se aleatoriamente 2 aves de cada box para serem abatidas. Estas constituíram a unidade experimental, e dessa forma foram pesadas individualmente e aneladas no pé direito. Após o abate a tíbia direita foi descarnada e levada para o refrigerador para ser posteriormente analisada. O teor de $\mathrm{Zn}$ foi calculado em relação ao peso da tíbia seca.

\section{Modelo experimental}

Utilizou-se o delineamento inteiramente ao acaso com 8 repetições, num esquema fatorial $2 \times 2 \times 9$ adaptado de Teixeira (1994). Este fatorial envolveu dois experimentos, dois sexos e nove doses de $\mathrm{Zn}(0,15,30,45,60$, 75, 90, 105, 120 ppm), sendo a variável estudada o teor de $\mathrm{Zn}$ na tíbia em ppm. A análise de variância foi realizada no software estatístico SISVAR (FERREIRA, 2000), considerando o seguinte modelo:

$$
Y_{i j k l}=\mu+E_{i}+S_{j}+D_{k}+(E S)_{i j}+(E D)_{i k}+(S D)_{j k}+(E S D)_{i j k}+e_{i j k l}
$$

$\operatorname{com} i=1,2 ; j=1,2 ; k=1,2,3,4,5,6,7,8,9 ; l=1,2,3,4,5,6,7,8$.

em que:

$Y_{i j k l}$ é o teor de Zn na tíbia em ppm da ave $l$, do $\operatorname{sexo} j$, do experimento $i$ que recebeu a dose $k$;

$\mu$ é uma constante associada a cada observação;

$E_{i}$ é o efeito do experimento $i ; S_{j}$ é o efeito do sexo $j$;

$D_{k}$ é o efeito da dose $k ;(E S)_{i j}$ é o efeito da interação entre experimento e sexo;
$(E D)_{i k}$ é o efeito da interação entre experimento e dose; $(S D)_{i k}$ é o efeito da interação entre sexo e dose;

$(E S D)_{i j k}$ é o efeito da interação entre experimento, sexo e dose;

$e_{i j k l}$ é o erro experimental associado a cada observação, independente e com distribuição normal de média zero e variância $\sigma^{2}$. 


\section{Modelos estatísticos ajustados}

O ajuste dos modelos de platô de resposta foi realizado considerando as médias estimadas pelo modelo (1) para o teor de $\mathrm{Zn}$ na tíbia referentes as doses de $\mathrm{Zn}$ estudadas, para posterior ajuste dos modelos por meio do procedimento NLIN (PROC NLIN) do sistema SAS Institute (1995).

i. Modelo Polinomial Quadrático com Resposta em Platô (MPQ)

Considerou-se as seguintes equações:

$$
\begin{gathered}
Y=a+b x+c x^{2}+e \quad \text { se } x<x_{0} \text { (quadrático) } \\
Y=p+e \quad \text { se } x \geq x_{0}, \quad \text { (platô) }
\end{gathered}
$$

em que:

$Y$ é o teor médio de $\mathrm{Zn}$ na tíbia referente a dose $x$ de $\mathrm{Zn}$. Dessa forma, para valores de $x$ menores que $x_{0}$, o modelo que descreve a resposta $Y$ é uma função quadrática, e para valores de $x$ maiores ou iguais a $x_{0}$, a equação é uma constante ou platô. Para estimação dos parâmetros, o modelo deve apresentar propriedades matemáticas adequadas, isto é, deve ser uma função contínua e diferenciável em $x_{0}$. Esta condição implica que: $x_{0}=-b / 2 c$ e $p=a-b^{2} / 4 c$, em que: $x_{0}$ é a dose máxima de Zn para uma máxima deposição de $\mathrm{Zn}$ do animal e o ponto de intercessão das duas linhas $p$ é o platô, sendo $a, b$ e $c$ os parâmetros do modelo a serem estimados.

ii. Modelo Não-Linear Exponencial 1 com Resposta em Platô (MNLE1)

Considerou-se o seguinte modelo apresentado por Rezende et al. (2000).

$$
Y=\operatorname{aexp}\left[-c(x-b)^{2}\right]+e
$$

se $x<x_{0}$ (exponencial)

$$
Y=p+e
$$

se $x \geq x_{0}, \quad$ (platô)

Da mesma forma que nos modelos (2) e (3), ao se derivar Y em relação a $x$, tem-se:

$$
\frac{\partial y}{\partial x}=\frac{\partial\left\{a \exp \left[-c(x-b)^{2}\right]\right\}}{\partial x}=a \exp \left[-c(x-b)^{2}\right][-2 c(x-b)]
$$

Igualando-se o resultado da derivação a zero e resolvendo-se para $x=x_{0}$, obtém-se

$x_{0}=b$. Substituindo-se $x$ pelo valor de $x_{0}$ na equação inicial, encontra-se

$$
p=f\left(x_{0}\right)=a \exp \left[-c(b-b)^{2}\right] \text {, resultando }
$$
em $p=a$, sendo $\mathrm{x}_{0}$ o estimador da dose máxima de $\mathrm{Zn}$, para uma máxima deposição de $\mathrm{Zn}$, o ponto de intercessão das duas linhas; $p$ é a estimador do platô, $a$, $b$ e $c$ são parâmetros do modelo a serem estimados.

Do ponto de vista da teoria da estimação, este modelo é considerado não-linear com relação a seus parâmetros, uma vez que as derivadas parciais dos parâmetros do segmento anterior ao ponto $x_{0}$, o platô e o próprio $x_{0}$ são funções dos próprios parâmetros. iii. Modelo Não-Linear Exponencial 2 com Resposta em Platô (MNLE2)

Considerou-se o modelo exponencial

$$
\begin{gathered}
Y=\operatorname{aexp}\left(b x-c x^{2}\right)+e \text { se } x<x_{0} \text { (exponencial) (6) } \\
Y=p+e \quad \text { se } x \geq x_{0} \text {, (platô) }
\end{gathered}
$$

em que $Y$ é o teor de $Z n$ do animal que recebeu a dose $x$ de Zn e $a, b, c$ são parâmetros do modelo.

Como apresentado nos modelos anteriores, a primeira derivada parcial deve ser idêntica no ponto $x=x_{0}$ para que a condição de continuidade das curvas antes e após o platô seja atendida. Assim, ao se derivar $y$ em relação a $x$, tem-se:

$$
\frac{\partial y}{\partial x}=\frac{\partial\left[\operatorname{aexp}\left(b x-c x^{2}\right)\right]}{\partial x}=a(b-2 c x) \exp \left(b x-c x^{2}\right)
$$


Igualando-se o resultado da derivação a zero e resolvendo-se para $x=x_{0}$, obtém-se

$x_{0}=b / 2 c$. Substituindo-se $x$ pelo valor de $x_{0}$ na equação inicial, encontra-se

$p=f\left(x_{0}\right)=a \exp \left[\frac{b^{2}}{2 c}-c\left(\frac{b^{2}}{4 c^{2}}\right)\right]$, que resulta em

$p=a \exp \left(\frac{b^{2}}{4 c}\right)$ em que $x_{0}$ é o estimador da dose máxima

de Zn, para uma máxima deposição de Zn, o ponto de intercessão das duas linhas; $p$ é a estimador do ponto do platô e $a, b, c$ são parâmetros do modelo a serem estimados.

\section{RESULTADOS E DISCUSSÃO}

Na Tabela 1 está apresentado o resumo da análise de variância.

TABELA 1 - Análise de variância e teste F para o teor de $\mathrm{Zn}$ em relação ao peso da tíbia seca.

\begin{tabular}{lccc}
\hline Fontes de Variação & GL & \multicolumn{2}{c}{ Quadrado Médio } \\
\hline Experimento & 1 & 32874,3261 & ${ }^{* *}$ \\
Sexo & 1 & 2375,0981 & $\mathrm{~ns}$ \\
Dose & 8 & 21607,3303 & $* *$ \\
Experimento $\times$ Sexo & 1 & 2629,9378 & $*$ \\
Experimento $\times$ Dose & 8 & 933,5284 & $\mathrm{~ns}$ \\
Sexo $\times$ Dose & 8 & 434,3318 & $\mathrm{~ns}$ \\
Experimento $\times$ Sexo $\times$ & 8 & 72,0330 & $\mathrm{~ns}$ \\
Dose & & & \\
Resíduo & 252 & 673,4048 & \\
\hline Total & 287 & Coeficiente de \\
& \multicolumn{3}{c}{ variação 9,83\% } \\
\hline
\end{tabular}

(**) significativo a $1 \%$ de probabilidade, $(*)$ significativo a $5 \%$ de probabilidade e (ns) não-significativo.
Nota-se pelos resultados do teste $F$ que houve significância para a fonte de variação Dose $(\mathrm{P}<0,01)$, sendo não-significativa as interações Sexo $\times$ Dose e Experimento Sexo Dose indicando que a resposta à dose de Zn independe do sexo e do experimento.

As médias do teor de $\mathrm{Zn}$ na tíbia, com erro padrão 4,5873, para cada dose de Zn estão apresentadas na Tabela 2.

As equações obtidas, com seus correspondentes Coeficientes de Determinação assintóticos $\left(\mathrm{R}^{2}\right)$, dose máxima de $\mathrm{Zn}\left(x_{0}\right)$ e platô $(p)$, estão apresentadas na Tabela 3 .

Pode-se notar pelos resultados apresentados na Tabela 3 que, os valores do coeficiente de determinação $\left(\mathrm{R}^{2}\right)$ foram elevados e bem próximos para os diferentes modelos. Carrijo \& Hochmuth (2000) e Cerrato \& Blackmer (1990) comentam que o $\mathrm{R}^{2}$ não é, isoladamente, um critério adequado para discussão de ajuste de modelos, pois, geralmente em ajuste de modelos não-lineares, é comum a obtenção de $\mathrm{R}^{2}$ assintóticos altos e similares.

Observa-se também que os modelos não-lineares foram semelhantes, uma vez que apresentaram praticamente a mesma estimativa para $\mathrm{x}_{0}$ e para $p$. Em relação à interpretação prática desses parâmetros, nas condições do presente trabalho, é possível inferir que a dose ideal de Zn na dieta de frangos de corte está próxima a 70 ppm a qual corresponde a um teor de Zn na tíbia próximo a 280 ppm.

Quanto à escolha do melhor modelo, é preferível optar pelo modelo não-linear 1 (MNLE1) devido à sua maior praticidade relacionada com a facilidade de interpretação de $\mathrm{x}_{0}$ e do platô, os quais correspondem, respectivamente, aos parâmetros b e a. O Modelo Polinomial Quadrático com platô (MPQ) apresentou um maior valor para $\mathrm{x}_{0}$ quando comparado com os modelos não-lineares. Este último resultado concorda com os obtidos por Coelho (1984), Euclydes \& Rostagno (2001), Morris (1983) e Runho et al. (2001), que afirmaram ter este modelo a característica de superestimar as exigências nutricionais do animal ou planta.

Na Tabela 4 está apresentado o teste de falta de ajuste para os três modelos estudados.

TABELA 2 - Médias estimadas pelo modelo (1) utilizadas no ajuste dos modelos de platô.

\begin{tabular}{cccc}
\hline Dose de Zn & Média observada & Dose de Zn & Média observada \\
\hline 0 & 208,97 & 75 & 280,49 \\
15 & 234,31 & 90 & 281,16 \\
30 & 257,47 & 105 & 282,84 \\
45 & 270,22 & 120 & 279,27 \\
60 & 280,03 & ------- & ---- \\
\hline
\end{tabular}

Ciênc. agrotec., Lavras, v. 31, n. 2, p. 468-478, mar./abr., 2007 
Os resultados apresentados na Tabela 4 comprovam que o teste $\mathrm{F}$ para os desvios de regressão foram não-significativos para todos os modelos, implicando na capacidade desses modelos em descrever os dados analisados, ou seja, não é rejeitada a hipótese dos modelos serem adequados.
Na Figura 2 estão representados os três modelos estudados.

Nota-se pela Figura 2 que todos os modelos apresentaram curvas muito semelhantes, havendo assim uma sobreposição que impede a identificação individual da dispersão gráfica de cada modelo.

TABELA 3 - Equações estimadas para os diferentes modelos, Coeficientes de Determinação assintóticos $\left(\mathrm{R}^{2}\right)$, dose máxima de $\mathrm{Zn}\left(x_{0}\right)$ e platô $(p)$.

\begin{tabular}{llccc}
\hline & \multicolumn{1}{c}{ Modelos } & $\mathbf{R}^{2}$ & $\boldsymbol{x}_{\mathbf{0}}$ & $\boldsymbol{p}$ \\
\hline MPQ & $Y=208,4413+2,0222 x-0,014077 x^{2}$ & 0,9979 & 71,8 & 281,1 \\
MNLE1 & $Y=280,9151 \exp \left[-0,00006372(x-68,1005)^{2}\right]$ & 0,9980 & 68,1 & 280,9 \\
MNLE2 & $Y=209,0390 \exp \left(0,008679 x-0,00006372 x^{2}\right)$ & 0,9980 & 68,1 & 280,9
\end{tabular}

TABELA 4 - Análise de variância e teste F, verificando a falta de ajuste para os modelos analisados.

\begin{tabular}{lcccc}
\hline & & \multicolumn{3}{c}{ Quadrado Médio } \\
\cline { 3 - 5 } Fonte de Variação & GL & MPQ & MNLE1 & MNLE2 \\
\hline Mod. Reg. não Corrigido & 3 & 6741401,4536 & 6741408,1630 & 6741408,1630 \\
Desvio de regressão & 6 & $59,5414^{\text {ns }}$ & $56,1867^{\text {ns }}$ & $56,1867^{\text {ns }}$ \\
Resíduo & 252 & 673,4048 & 673,4048 & 673,4048 \\
\hline
\end{tabular}

${ }^{\mathrm{ns}}$ não-significativo pelo teste $\mathrm{F}$.

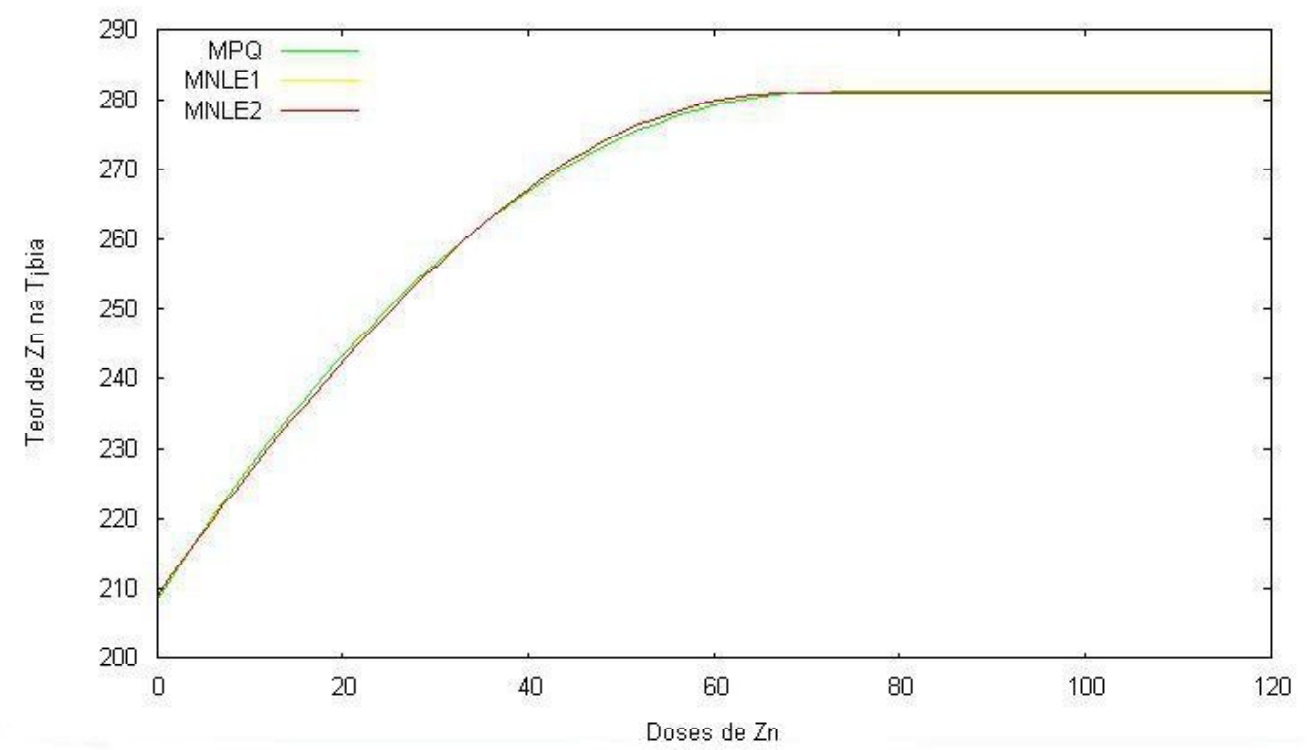

FIGURA 2 - Teor de Zn na tíbia de frangos de corte em função das doses de Zn na ração, para os três modelos estudados. 


\section{CONCLUSÕES}

Todos os modelos avaliados mostraram-se adequados ao estudar a exigência de Zinco em frangos de corte, sendo impossível indicar aquele de maior qualidade.

O modelo não-linear 1 (MNLE1) é recomendado por ser de utilização mais prática, uma vez que fornece diretamente a dose máxima de $\mathrm{Zn}$ e o ponto inicial do platô de resposta.

\section{REFERÊNCIAS BIBLIOGRÁFICAS}

BAKER, D. H. Problems and pitfalls in animal experiments designed to establish dietary requirements for essential nutrients. Journal of Nutrition, Cambridge, v. 116, p. 23392349, 1986.

BRAGA, J. M. Avaliação da fertilidade do solo: ensaios de campo. Viçosa: UFV, 1983. 101 p.

BULLOCK, D. G.; BULLOCK, D. S. Quadratic and quadraticplus-plateau models for predicting optimal nitrogen rate of corn: a comparison. Agronomy Journal, Madison, v. 86, p. 191-195, 1994.

CARRIJO, O. A.; HOCHMUTH, G. Estimativa do rendimento do tomateiro usando modelos estatísticos com teores iniciais de fósforo no solo e níveis de adubação fosfatada: pesquisa em andamento no 19 Embrapa/CNPH. Disponível em: <http:// iwww.cnph.embrapa.br/pa/pa19.html set. 2000 .

CELLA, P. S.; DONZELE, J. L.; OLIVEIRA, R. F. M.; ALBINO, L. F. T.; FERREIRA, A. S.; GOMES, P. C.; VALERIO, S. R.; APOLONIO, L. R. Níveis de lisina mantendo a relação aminoacídica para frangos de corte no período de 1 a 21 dias de idade, em diferentes ambientes térmicos. Revista Brasileira de Zootecnia, Viçosa, v. 30, n. 2, p. 433-439, 2001.

CERRATO, M. E.; BLACKMER, A. M. Comparison of models for describing corn yields response to nitrogen fertilizer. Agronomy Journal, Madison, v. 82, p. 138143, 1990.

COELHO, L. S. S. Exigências de proteína e lisina para suínos de 15 a 30kg de peso vivo. 1984. 139 f. Tese (Doutorado em Zootecnia) - Universidade Federal de Viçosa, Viçosa, 1984.
COELHO, L. S. S.; COSTA, P. M. A.; SILVA, M. A.; PEREIRA, J. A. A.; ROSTAGNO, H. S.; BARBOSA, H. P. Modelos para estimar exigências nutricionais em suínos. Revista da Sociedade Brasileira de Zootecnia, Viçosa, v. 16, n. 1, p. 102-110, 1987.

EUCLYDES, R. F.; ROSTAGNO, H. S. Estimativa dos níveis nutricionais via experimentos de desempenho [mensagem pessoal]. Texto enviado por <rostagno@ufv.br> em 20 set. 2001. Palestra proferida no Workshop Latino Americano Anjinomoto Biolatina, em Foz do Iguaçu, 18-20 de junho de 2001.

FERREIRA, D. F. Análises estatísticas por meio do SISVAR para Windows versão 4.0. In: REUNIÃO ANUAL DA REGIÃO BRASILEIRA DA SOCIEDADE INTERNACIONAL DE BIOMETRIA (RBRAS), 45., 2000, São Carlos, SP. Anais... São Carlos: UFSCar, 2000. p. 255-258.

FIGUEIREDO, D. F.; TORAL, F. L. B.; GARCIA, J.; MURAKAMI, A. E.; FURLAN, A. C. Níveis nutricionais de sódio para poedeiras pós-muda forçada. In: REUNIÃO ANUAL DA SOCIEDADE BRASILEIRA DE ZOOTECNIA, 38., 2001, Piracicaba. Anais... Piracicaba: FEALQ, 2001. p. 841-843.

GOMES, P. C.; GOMES, M. F. M.; ALBINO, L. F. T.; FIALHO, F. B.; LIMA, G. J. M. M. de; FIGUEIREDO, E. A. P. Exigência de fósforo disponível para frangos de corte nas fases de crescimento e terminação. Revista da Sociedade Brasileira de Zootecnia, Viçosa, v. 23, n. 4, p. 615-622, 1994.

MORRIS, T. R. The interpretation of response data from animal feeding trials. In: HARESIGN, W. Recent advances in animal nutrition. London: Butterworths, 1983. p. 13-23.

OLIVEIRA, A. L. S.; DONZELE, J. L.; OLIVEIRA, R. F. M.; FERREIRA, A. S.; COTA, T. S.; GENEROSO, R. R. Níveis de lisina para suínos machos castrados de alto potencial genético para deposição de carne magra dos 95 aos $110 \mathrm{~kg}$. In: REUNIÃO ANUAL DA SOCIEDADE BRASILEIRA DE ZOOTECNIA, 38., 2001, Piracicaba. Anais... Piracicaba: FEALQ, 2001.p. 817-819.

PACK, M. Models used to estimate nutrient requirements with emphasis on economic aspects. In: INTERNATIONAL SYMPOSIUM ON NUTRITIONAL REQUIREMENTS OF POULTRY AND SWINE; SIMPÓSIO INTERNACIONAL SOBRE EXIGÊNCIAS NUTRICIONAIS DE AVES E SUÍNOS, 1996, Viçosa, MG. Anais... Viçosa: UFV, 1996. p. 43-54. 
REZENDE, D. M. L. C.; MUNIZ, J. A.; FERREIRA, D. F. Proposição de um modelo não linear com response plateau. In: REUNIÃO ANUAL DA REGIÃO BRASILEIRA DA SOCIEDADE INTERNACIONAL DE BIOMETRIA (RBRAS), 45., 2000, São Carlos, SP. Anais... São Carlos: UFSCar, 2000. p. 96-99.

ROBBINS, K. R.; NORTON, H. W.; BAKER, D. H. Estimation of nutrient requirements from growth data. Journal of Nutrition, Baltimore, v. 109, p. 1710-1714, 1979.

RUNHO, R. C.; GOMES, P. C.; ROSTAGNO, H. S.; ALBINO, L. F. T.; LOPES, P. S.; POZZA, P. C. Exigência de fósforo disponível para frangos de corte machos e fêmeas de 1 a
21 dias de idade. Revista Brasileira de Zootecnia, Viçosa, v. 30, n. 1, p. 187-196, 2001.

SAS INSTITUTE. SAS STAT user's guide: version 6. 4. ed. Cary, 1989. v. 2.

SAS INSTITUTE. SAS language and procedures: usage. Version 6. Cary, 1995. 373 p.

TEIXEIRA, A. S. Exigências nutricionais de zinco e sua biodisponibilidade em sulfatos e óxidos de zinco para pintos de corte. 1994. 172 f. Tese (Doutorado em Desempenho Animal) - Universidade Federal do Rio Grande do Sul, Porto Alegre, 1994. 


\section{ANEXO (ROTINAS SAS $\left.{ }^{\oplus}\right)$}

No Quadro 1 está apresentada a rotina para a estimação dos parâmetros $a, b, c$, do ponto $x_{0}$ e do platô por meio do modelo MPQ para a variável teor de Zn na tíbia em ppm. Esta rotina para o MPQ está apresentada nas páginas 1162 a 1165 do manual do SAS Institute (1989).

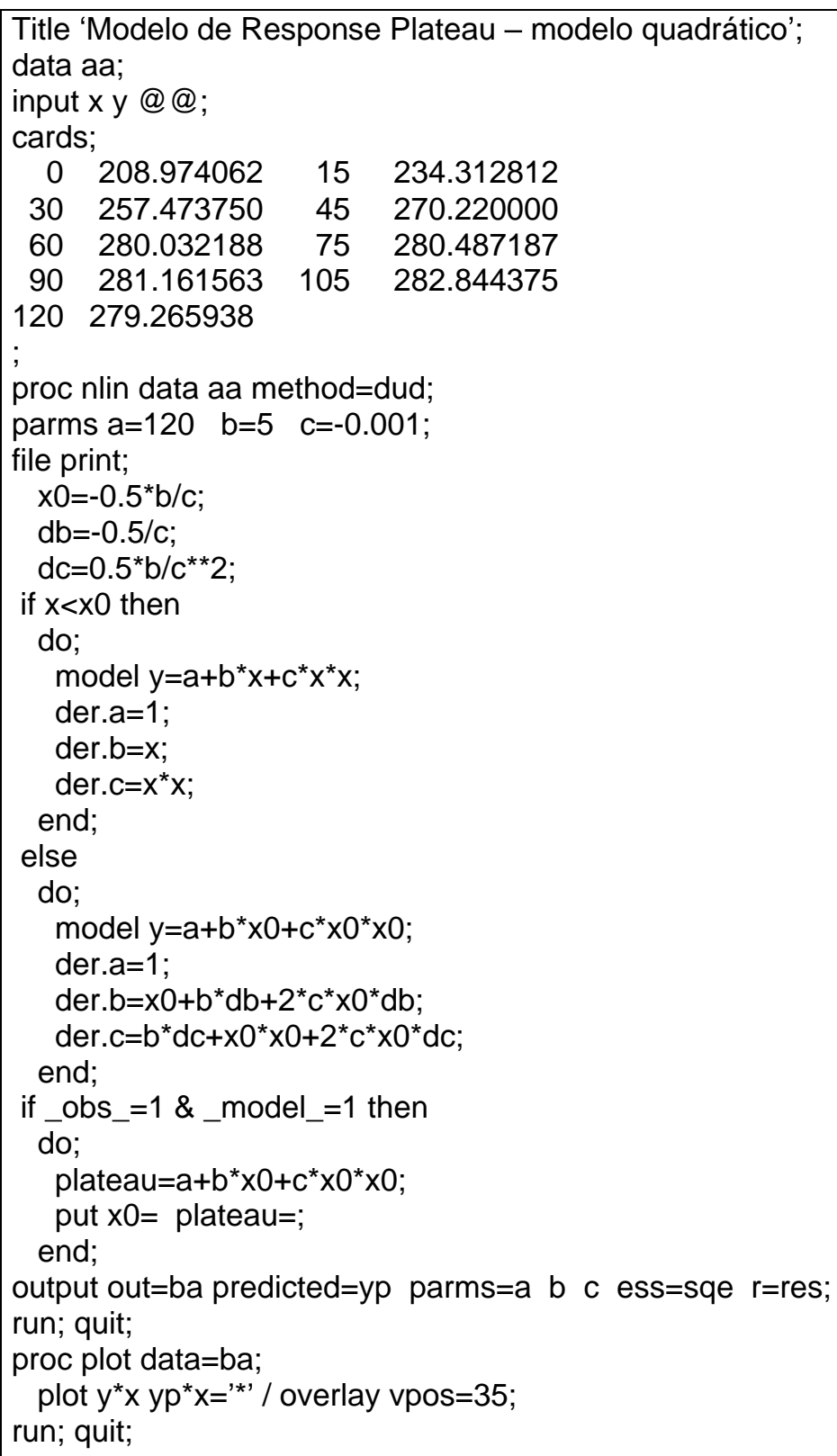

QUADRO 1 - Rotina $\mathrm{SAS}^{\circledR}$ para o MPQ. 
No Quadro 2 está apresentada a rotina para a estimação dos parâmetros $a, b, c$, do ponto $x_{0}$ e do platô por meio do modelo MNLE1 para a variável teor de Zn na tíbia em ppm.

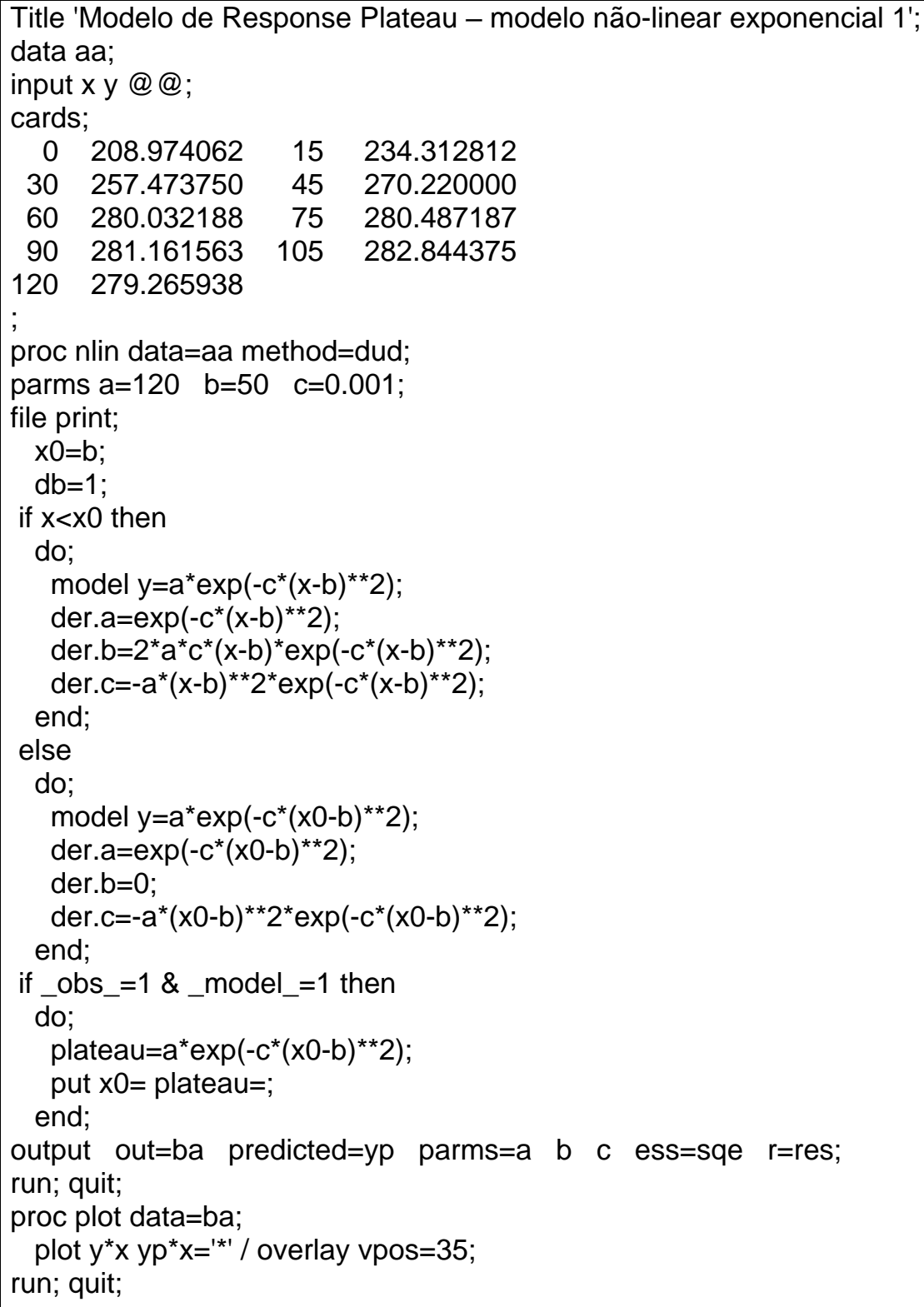

QUADRO 2 - Rotina SAS ${ }^{\circledR}$ para o MNLE1. 
No Quadro 3 está apresentada a rotina para a estimação dos parâmetros $a, b$, do ponto $x_{0}$ e do platô por meio do modelo MNLE2 para a variável teor de Zn na tíbia em ppm.

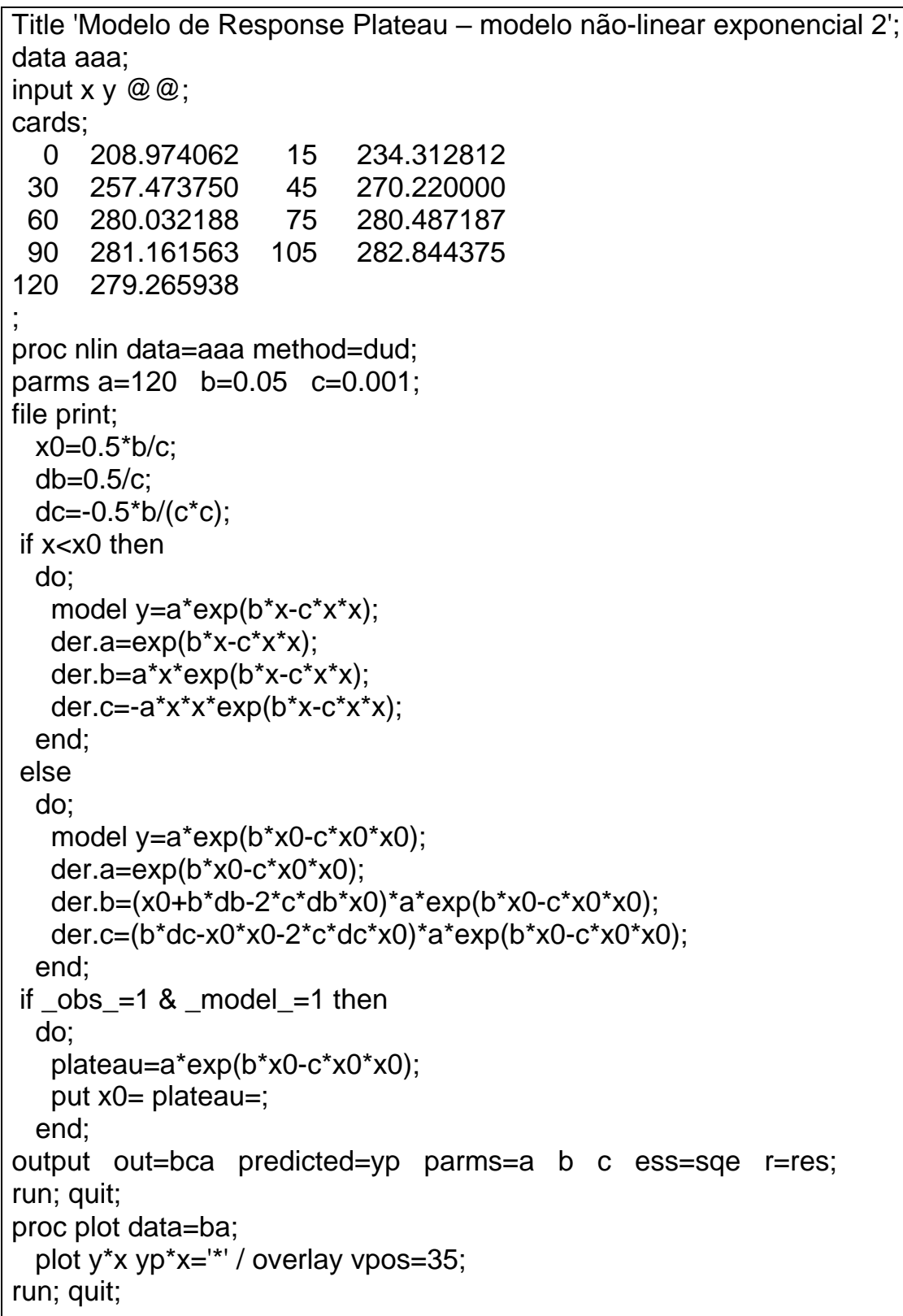

QUADRO 3 - Rotina SAS ${ }^{\circledR}$ para o MNLE2. 